\title{
AN OBSTRUCTION TO EMBEDDING 4-TANGLES IN LINKS
}

\author{
DAVID A. KREBES
}

\begin{abstract}
We consider the ways in which a 4-tangle $T$ inside a unit cube can be extended outside the cube into a knot or link $L$. We present two links $n(T)$ and $d(T)$ such that the greatest common divisor of the determinants of these two links always divides the determinant of the $\operatorname{link} L$.

In order to prove this result we give a two-integer invariant of 4tangles. Calculations are facilitated by viewing the determinant as the Kauffman bracket at a fourth root of -1 , which sets the loop factor to zero. For rational tangles, our invariant coincides with the value of the associated continued fraction.
\end{abstract}

\section{INTRODUCTION}

In this article, we will consider the ways in which a knot or link $L$ can intersect a ball $B$ in $\mathbf{R}^{\mathbf{3}}$. We examine the case in which $L$ meets the boundary of $B$ transversely in four points. The pair $(B, L \cap B)$ is called a 4-tangle (or simply tangle) and is said to sit inside or be embedded in the link $L$. Section 2 describes tangles. (Note that the definition of tangle which we will use here is slightly more general than that found in [3], which deals only with what we will call loop-free tangles.)

The results proven in this paper involve the determinant of a knot or link $L$, which is a non-negative integer denoted $|\langle L\rangle|$. (This notation

This paper was prepared while the author was a graduate student at the University of Illinois at Chicago, a Pacific Institute for the Mathematical Sciences post-doctoral fellow at the University of British Columbia, and a visiting assistant professor at the University of South Alabama. 


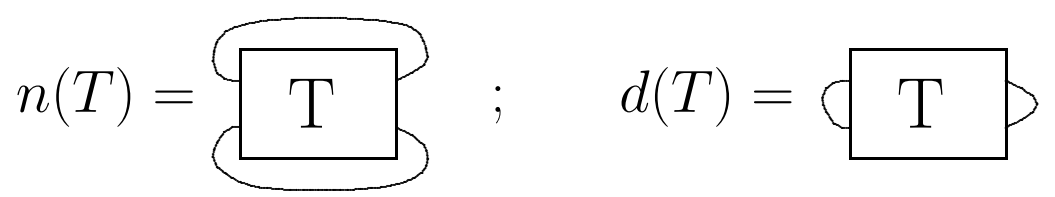

Figure 1. Two important links in which the tangle $T$ embeds has a meaning which will be explained later in this introduction; see also Section 11.)

We are interested in the ways a tangle $T$ can be embedded in a link. Two such embeddings are given in Figure 1.

The main result of our paper is the following:

Theorem 1 (Version 2). If the tangle $T$ can be embedded in the link $L$, then the greatest common divisor of the determinants of $n(T)$ and of $d(T)$ divides the determinant of $L$.

Since the determinant of the unknot is 1 , we have the following corollary.

Corollary 1. The tangle $T$ cannot sit inside the unknot unless the determinants of $n(T)$ and $d(T)$ are relatively prime.

Remark 1. Corollary 1 can also be regarded as a knotting criterion: If $K \cong S^{1} \subset S^{3}$ has a tangle $T$ embedded in it such that the determinants of $n(T)$ and $d(T)$ are not relatively prime, then $K$ is knotted.

The following claim illustrates Corollary 1: If a closed curve intersects the unit cube (a topological ball) in the tangle $T$ shown on the left in Figure 2 ( $T$ is known to laypeople as a square knot), then it is genuinely knotted.

For example the curve depicted on the right in Figure 2 is knotted: A rubber band, for instance, cannot be manipulated into this shape without breaking it and gluing the ends back together. The determinant of $n(T)$, a connected sum of two trefoils, is 9 and the determinant of $d(T)$, the unlink 

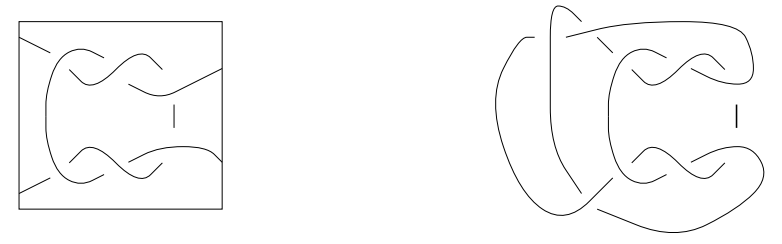

Figure 2. The square tangle and a knot in which it sits

of two components, is 0 . Since 9 and 0 are not relatively prime, Corollary 1 may be applied, proving the claims.

The particulars of this example are contained in Section 10. An alternative proof for the reader familiar with Fox $n$-colourings is found in Section 13.

To prove Theorem 1, Version 2, we introduce an invariant of tangles whose values are formal fractions $p / q$, not necessarily reduced, as defined in Section 6. A precise definition of the invariant appears in Section 7. However, it is rather easy to define $p$ and $q$ up to sign:

Proposition 4. If $f(T)=p / q$ then $|p|$ and $|q|$ are the determinants $|\langle n(T)\rangle|$ and $|\langle d(T)\rangle|$, respectively.

Thus Theorem 1, Version 2 is equivalent to:

Theorem 1, Version 1. If $T$ is a tangle with invariant $p / q$ and $T$ can be embedded in the link $L$, then $\operatorname{gcd}(p, q)$ divides $|\langle L\rangle|$.

To define this invariant we consider the Kauffman bracket, described in [1], evaluated at an eighth root of unity, namely $A=e^{\pi i / 4}$ where $A$ is the indeterminate in the Kauffman bracket polynomial. Some consequences of this choice are described in Section 4. With this choice of $A$ the absolute value $|\langle L\rangle|$ of the Kauffman bracket is precisely the determinant of the knot or link $L$.

Our invariant has an agreeable additivity property: 
Proposition 5. (paraphrase). If $p / q$ is the value of the invariant on the tangle $T$, and $r / s$ is the value of the invariant on the tangle $T^{\prime}$, then the value of the invariant on the tangle $T+T^{\prime}$, which is described in Section 2, is

$$
\frac{p s+q r}{q s}
$$

This last expression is seen to be the formal sum of $p / q$ and $r / s$ when the product of the denominators is used as a common denominator in elementary school addition of fractions.

Proposition 5 is used in conjunction with the following:

Lemma 5. If the tangle $T$ sits inside the link $L$, then there is a second tangle $T^{\prime}$ such that $L$ is ambient isotopic to the link $n\left(T+T^{\prime}\right)$.

Thus if $p / q$ is the value of the invariant on the tangle $T$ then there are integers $r$ and $s$ such that the determinant of the link $L$ is $|p s+q r|$. Thus any common divisor of $p$ and $q$ divides the determinant of $L$, and Theorem 1 is established.

In Section 12 we illustrate the power of the invariant by showing that any fraction $p / q$ (not necessarily reduced) is associated to at least one tangle. If $p$ or $q$ is odd, this tangle may be chosen to consist of two unknotted $\operatorname{arcs}$ in $B$. (It is loop-free by Proposition 9.)

A by-product of this investigation is an efficient way of calculating the determinants of a group of links systematically using the Kauffman bracket. See Section 11.

\section{4-TANGLES}

All our work will be done in the PL (piecewise-linear) category.

Consider a unit cube $J=I^{3}$ (here $I$ is the unit interval $[0,1]$ of the real line) and a one-dimensional properly embedded submanifold $T$ such that $\partial T=T \cap \partial J$ consists of four points. Clearly $T$ is homeomorphic to the disjoint union $I \coprod I \coprod S^{1} \coprod \ldots \amalg S^{1}$ of two unit intervals and some number 

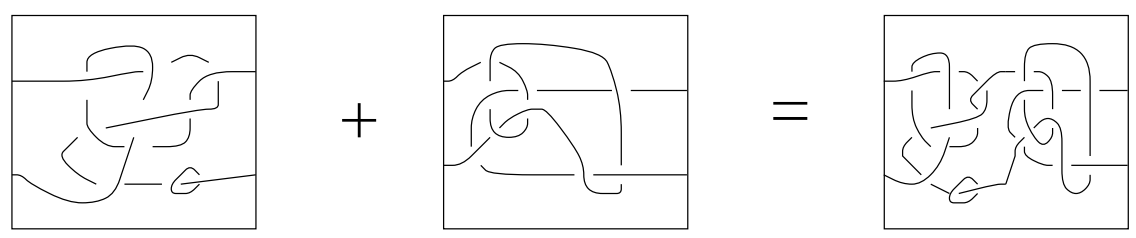

Figure 3. The addition rule for tangles

(possibly zero) of circles. There is an isotopy of $(J, T)$ which takes $\partial T$ to the four points

$$
\left(0, \frac{1}{3}, \frac{1}{2}\right), \quad\left(0, \frac{2}{3}, \frac{1}{2}\right), \quad\left(1, \frac{1}{3}, \frac{1}{2}\right), \quad\left(1, \frac{2}{3}, \frac{1}{2}\right)
$$

of $\partial J$. Let us therefore assume that $\partial T$ consists of these four points.

In this paper $J$ will be drawn as projected along the z-axis to the square $I \times I \times\{1 / 2\}$. See diagrams.

Since $T$ is homeomorphically a disjoint union it is a disconnected space and we may refer to its components; the two components homeomorphic to the unit interval $I$ will be called the arc components and the components homeomorphic to $S^{1}$ will be called loops or sometimes cycles. When there are no loops the two arc components will be referred to simply as strands.

The set of such pairs $(J, T)$ has a monoid structure, where addition is obtained by horizontal concatenation and compression, eg. see Figure 3.

Formally, $(J, T)+\left(J, T^{\prime}\right)=\left(J, \Lambda(T) \cup \Psi\left(T^{\prime}\right)\right)$ where

$$
\begin{gathered}
\Lambda: J \rightarrow J:(x, y, z) \rightarrow\left(\frac{1}{2} x, y, z\right) \\
\Psi: J \rightarrow J:(x, y, z) \rightarrow\left(\frac{1}{2} x+\frac{1}{2}, y, z\right)
\end{gathered}
$$

Finally, a 4-tangle (tangle) will be an ambient isotopy (deformations without double points and with fixed endpoints) class of such pairs $(J, T)$. The above-mentioned monoid structure on the space of pairs induces a monoid structure on the space $\mathbf{T}$ of 4 -tangles which will be denoted 
"+", and the tangle $T+T^{\prime}$ will be called the sum of $T$ and $T^{\prime}$. The reader should recognize, however, that this monoid structure is not commutative.

\section{Diagrams, Shadows, and States}

Define a tangle shadow, like a link shadow (see [5]) as the underlying combinatorial object obtained from a tangle diagram by disregarding which is the over- and which is the undercrossing arc at each crossing. Link shadows can be thought of as planar graphs, where each vertex is incident to four edges.

By a component of a tangle shadow we shall mean the part corresponding to a component of any tangle which projects to the given shadow. In other words, a component is a minimal non-empty collection of edges where the edges opposite each other at a vertex are considered to belong to the same component.

As in the literature (eg. [1]) we will use the term state to refer both to a smoothing at a particular crossing and to a choice of such smoothings over all crossings of a tangle or link. If we choose a state for each crossing of a tangle or link diagram then we arrive at a new tangle or link diagram- one without crossings- which in turn represents a (rather trivial) tangle or link. Thus we may also talk about components of tangle or link states. With this in mind we make the following definition.

Definition 1. Define a tangle, tangle diagram, tangle shadow or tangle state to be loop-free or acyclic if it has only the two arc components and no loops (like the tangles in [3]). Two acyclic tangles, tangle diagrams, tangle shadows or tangle states will be said to be homotopic to one another if the strands pair off the endpoints in the same way; equivalently, if when one starts at the upper left hand endpoint and follows the strand originating from there one arrives at the same endpoint in either case. 


\section{The Kauffman Bracket at an Eighth Root of Unity}

See [1] for an exposition of the Kauffman bracket, regular isotopy and the Reidemeister moves.

Notation 1. Throughout this paper the expression $\langle L\rangle$ will always mean the Kauffman bracket for the regular isotopy class $L$ of link diagrams evaluated at

$$
A=e^{\pi i / 4}
$$

Notation 2. Let $\Phi=\left\{u \mid u^{8}=1\right\} \subset \mathbf{C}$, the 8th roots of unity, or powers of A.

Definition 2. A monocyclic state for a link or a link shadow is a state which consists of a single loop. Monocyclic states are the only states of interest to us since they are the only states on which the Kauffman bracket evaluated at $A=e^{\pi i / 4}$ is non-zero. This is because second, third or subsequent loops each introduce the factor $-A^{2}-A^{-2}$ which evaluates to $-i-\left(i^{-1}\right)=-i--i=0$.

Lemma 1. Let $L$ and $L^{\prime}$ be two link diagrams which differ by a single Reidemeister move. If this move is of type II or III then $\langle L\rangle=\left\langle L^{\prime}\right\rangle$. If the move is of type $I$ then $\langle L\rangle=A^{ \pm 1}\left\langle L^{\prime}\right\rangle$.

Proof. Invariance under type II or III moves is the meaning of the statement that the Kauffman bracket is an invariant of regular isotopy (see $[1])$.

Behaviour under one version of the Type I Reidemeister move is given in equation (1); the calculations for the other versions (the kink may protrude to the left for instance or the roles of the over- and undercrossing arcs may be reversed from that shown on the left hand side of equation (1)) are similar. 


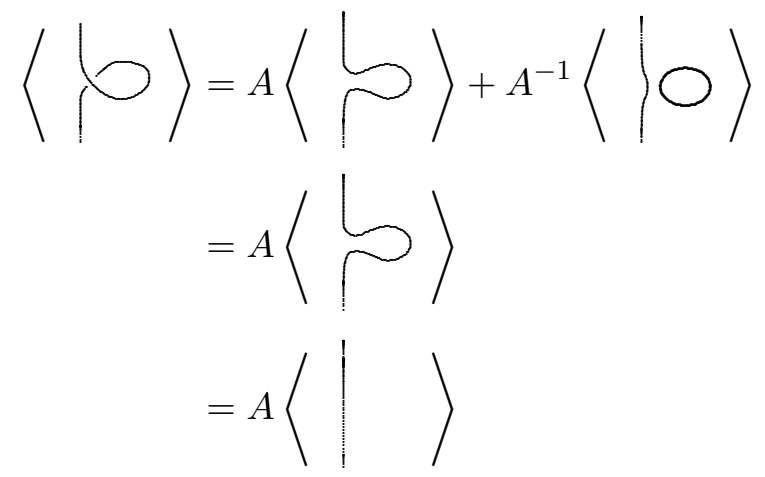

Equation (1) completes the proof of Lemma 1.

Lemma 2. Any two monocyclic states for a link diagram $L$ (or for a link shadow) differ at an even number of crossings of the original diagram.

Proof. Consider the checkerboard shading (see [5]) for the link diagram $L$, thought of as drawn on $S^{2}$. This divides $S^{2}$ into shaded and unshaded regions, which we can think of as the vertices of a graph. A state for $L$ connects these regions in various ways, ie. forms edges between the vertices. The resulting planar graph is the union of two subgraphs: the "shaded" one and the "unshaded" one. Note that a loop of one of these graphs bounds, on either side, at least one component of the other graph.

Now any component of either graph is bounded by a cycle of the state. So monocyclic states induce graphs with only two components: the shaded one and the unshaded one. It follows that these graphs are both trees. Hence if there were $k$ shaded regions of the shadow, there must be exactly $k-1$ crossings which are resolved shaded-to-shaded in a monocyclic state (since in any finite tree there is one more node than edges). So if we replace one monocyclic state by another then there are as many crossings whose resolution changes from unshaded-to-unshaded to shaded-to-shaded as crossings whose resolution changes in the other direction. Hence there are in total an even number of crossings whose resolution changes, proving Lemma 2. 
Proposition 1. Let $L$ be any link diagram. Then $\langle L\rangle \in \mathbf{Z} \cdot \Phi$, the complex numbers of the form pu, where $p \in \mathbf{Z}$ and $u \in \Phi$. In particular $|\langle L\rangle|$ is a non-negative integer.

Proof. Consider two monocyclic states for $L$. By Lemma 2 these states disagree at an even number of crossings. Since each change of state at a crossing introduces a factor of $A^{2}$ or $A^{-2}$, an even number of changes of state introduces a factor which is a power of $A^{4}$ which evaluates to -1 .

Now if $\langle L\rangle \neq 0$ then there is a monocyclic state whose coefficient in the Kauffman bracket is a power of $A=e^{\pi i / 4} \in \Phi$; say it is $u \in \Phi$. Then any other monocyclic state has coefficient $\pm u$. Adding together these state values we obtain the value for $\langle L\rangle$ of $p u$ where $p \in \mathbf{Z}$. This is Proposition 1 .

The integer $|\langle L\rangle|$ is known classically as the determinant of the link L. See Section 11. It is an invariant of ambient isotopy:

Proposition 2. $|\langle L\rangle|$ depends only on the ambient isotopy class of the link diagram $L$.

Proof. We need only check that $|\langle L\rangle|$ remains invariant under each of the three types of Reidemeister moves. See Lemma 1. Reidemeister Move Type I multiplies $\langle L\rangle$ by $A^{ \pm 1}=e^{ \pm \pi i / 4}$ and so leaves the absolute value of the bracket invariant. Reidemeister Moves of type II and III leave the bracket, and therefore its absolute value, unchanged. Proposition 2 follows.

Remark 2. In view of Proposition 2 we will allow ourselves to consider the expression $|\langle L\rangle|$ where $L$ is an ambient isotopy class of links or link diagrams, even though $\langle L\rangle$ is only defined on regular isotopy classes of link diagrams.

\section{Numerator and Denominator}

Let $T$ be a tangle. We wish to consider what sort of links the tangle $T$ can sit inside. Two such links are $n(T)$ and $d(T)$, depicted in Figure 1. If $T$ is a tangle diagram, then $n(T)$ and $d(T)$ are link diagrams. Since the crossings of $n(T)$ and of $d(T)$ are precisely the crossings of $T$, states for 

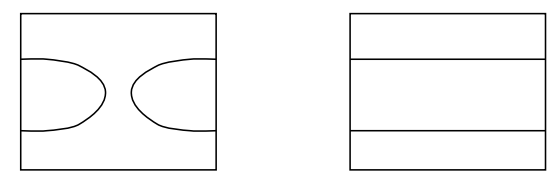

Figure 4. Numerator and denominator states

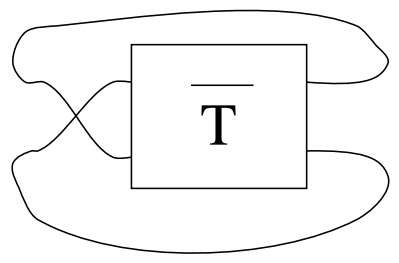

Figure 5. A link shadow based on the tangle shadow $\bar{T}$

$T$ correspond to states for $n(T)$ and to states for $d(T)$. There are only two homotopy types of acyclic tangle states: the numerator states and the denominator states, as shown in Figure 4. Numerator states for $T$ are then precisely the states which correspond to monocyclic states for $n(T)$ and thus are precisely the states which contribute to $\langle n(T)\rangle$ :

$$
\langle n(T)\rangle=\sum_{\substack{\text { numerator } \\ \text { states for } T}} \prod_{\text {vertices }} A^{ \pm 1}
$$

and similarly for $\langle d(T)\rangle$ and denominator states. Here by "vertices" we mean 4-vertices of the original tangle shadow; ie. crossings of the tangle; for a given state at a given crossing we choose $A$ or $A^{-1}$ according to the skein relation for the Kauffman bracket (see [1]).

Lemma 3. Consider a tangle $T$. Any numerator state for $T$ differs from any denominator state for $T$ at an odd number of crossings.

Proof. Consider the link shadow $L$ given in Figure 5 , where $\bar{T}$ is the shadow of $T$.

For any numerator (denominator respectively) state for $T$ we have that the state of Figure 6 on the left (on the right respectively) is monocyclic. (The portion of the state inside the box is shown up to homotopy.) By 

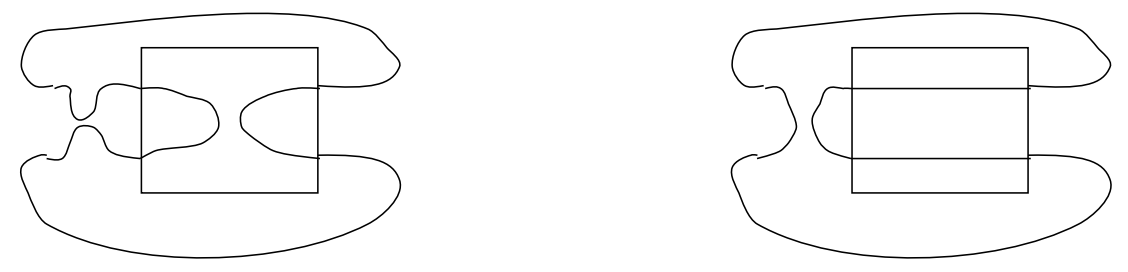

Figure 6. Two monocyclic link states

Lemma 2 these states for L differ at an even number of crossings. Since they differ at one crossing outside the box they must differ at an odd number of crossings inside the box. Hence any numerator state for $\mathrm{T}$ differs from any denominator state for $\mathrm{T}$ at an odd number of crossings. This is Lemma 3.

Proposition 3. If $\langle n(T)\rangle=p u$ and $\langle d(T)\rangle=q v$, with $p, q \in \mathbf{Z}$ and $u, v \in$ $\Phi$, then at least one of the following four equalities holds:

$$
p=0, q=0, \frac{u}{v}=i, \frac{u}{v}=-i
$$

Proof. If $\langle n(T)\rangle=0$ then $p u=0$ implies that $p=0$; if $\langle d(T)\rangle=0$ then $q v=0$ implies that $q=0$. In either case we are done. If on the other hand both $\langle n(T)\rangle$ and $\langle d(T)\rangle$ are non-zero then there is a numerator state of coefficient, say, $u^{\prime}$, and a denominator state with coefficient, say, $v^{\prime}$, where $u^{\prime}, v^{\prime} \in \Phi$. By Lemma 3 these states differ at an odd number of crossings. Since each crossing change contributes a factor of $A^{2}=i$ or $A^{-2}=-i$, an odd number of crossing changes contributes a factor of $\pm i$, and we have $u^{\prime} / v^{\prime}= \pm i$. Following the proof of Proposition $1,\langle n(T)\rangle$ is of form $p^{\prime} u^{\prime}$ for $p^{\prime} \in \mathbf{Z}$. We can assume $p \neq 0$. Then $p u=p^{\prime} u^{\prime}$ implies that $p^{\prime} / p=u / u^{\prime} \in \mathbf{Q} \cap \Phi=\{ \pm 1\}$, or $u= \pm u^{\prime}$. Similarly $v= \pm v^{\prime}$. Thus $u / v= \pm u^{\prime} / v^{\prime}= \pm( \pm i)= \pm i$. Proposition 3 follows.

\section{Formal Fractions}

The invariant of tangles $f$ which we will define in the next section takes values that are formal fractions, that is, formal quotients of two 
integers. Such fractions need not be reduced and can have zero as their denominators. We give a precise definition of such fractions below.

We define an equivalence relation $\sim$ on the collection $\mathbf{Z} \times \mathbf{Z}$ of ordered pairs of integers as follows:

$$
(p, q) \sim\left(p^{\prime}, q^{\prime}\right) \text { if }(p, q)=\left(p^{\prime}, q^{\prime}\right) \text { or }(p, q)=\left(-p^{\prime},-q^{\prime}\right) .
$$

Let us denote the set of equivalence classes as $\mathbf{A}$. We will use the notation $[p, q]$ or $p / q$ for the equivalence class containing the ordered pair $(p, q)$. In regards to the parallel with rational numbers, we may think of elements of $\mathbf{A}$ as fractions which are not allowed to be reduced except by the factor -1 . Thus $2 / 4 \neq 1 / 2$, but $1 / 2=-1 /-2$ and $3 / 0=-3 / 0$.

We can make $\mathbf{A}$ a monoid by the rule

$$
\frac{p}{q}+\frac{r}{s}=\frac{p s+q r}{q s}
$$

as we do with fractions except that for the common denominator we always use the product.

A straightforward calculation shows that this monoid structure on $\mathbf{A}$ is associative even when some of the denominators are zero.

Given the element $[p, q] \in \mathbf{A}$, we will frequently speak of properties of $p$ and $q$ for properties of the type that, if held by the pair $(a, b)$, are also held by the pair $(-a,-b)$. Thus it makes sense to say that, eg. " $q$ is odd," "the $\operatorname{gcd}$ of $p$ and $q$ is $d$," and " $p$ is zero."

\section{Definition of the Invariant}

In this section we will define a map $f$ from the space $\mathbf{T}$ of tangles to the space $\mathbf{A}$ of formal fractions.

Notation 3. For a tangle diagram $T$ define the set

$$
\Psi(T)=\{(u\langle n(T)\rangle, u i\langle d(T)\rangle) \mid u \in \Phi\} \subset \mathbf{C}^{2} .
$$

Lemma 4. The set $\Psi(T)$ depends only on the ambient isotopy class of the tangle diagram $T$. 
Proof. We must check that $\Psi(T)$ is invariant under each of the Reidemeister moves, for which we refer to Lemma 1. A Reidemeister move on the tangle $T$ corresponds to a Reidemeister move on $n(T)$ and to a Reidemeister move on $d(T)$. If this move is of type II or III the bracket evaluated at these links is unaffected; thus $\Psi(T)$ remains invariant as claimed.

If this move is of type I, then $\langle n(T)\rangle$ and $\langle d(T)\rangle$ are both multiplied by the same element $A^{ \pm 1} \in \Phi$. Thus the elements of $\Psi(T)$ are permuted and the set itself is left unchanged. This establishes Lemma 4.

In view of Lemma 4 we will refer to $\Psi(T)$ for tangles $T$ with no particular choice of diagram.

Theorem-and-Definition 1. Let $T \in \mathbf{T}$ be a tangle. Then $\Psi(T) \cap \mathbf{Z}^{2}$ is a set of the form $\{(p, q),(-p,-q)\}$. Thus we may regard $\Psi(T) \cap \mathbf{Z}^{2}$ as an element $[p, q]$ of the set $\mathbf{A}$ of formal fractions defined in Section 6. This defines a function $f: \mathbf{T} \rightarrow \mathbf{A}$.

Proof. Let $T$ be a fixed tangle. We must prove three items:

1. $f(T)=\Psi(T) \cap \mathbf{Z}^{2}$ is non-empty.

2. If both $(p, q)$ and $\left(p^{\prime}, q^{\prime}\right)$ are in $f(T)=\Psi(T) \cap \mathbf{Z}^{2}$ then $[p, q]=\left[p^{\prime}, q^{\prime}\right]$.

3. If $[p, q]=\left[p^{\prime}, q^{\prime}\right]$ and $(p, q) \in f(T)$ then $\left(p^{\prime}, q^{\prime}\right) \in f(T)$.

Proof of 1: Let $\hat{T}$ be a diagram of $T$. From Proposition 1 let $\langle n(\hat{T})\rangle=$ $p u$ and $\langle d(\hat{T})\rangle=q v$. From Proposition 3 we have $p=0, q=0, u / v=i$ or $u / v=-i$.

- Case $p=0$. Set $u^{\prime}=-i \bar{v}$. Then $u^{\prime}\langle n(\hat{T})\rangle=u^{\prime} \cdot 0=0 \in \mathbf{Z}$ and $u^{\prime} i\langle d(T)\rangle=-i \bar{v} i q v=q \in \mathbf{Z}$. Thus $(0, q) \in \Psi(T) \cap \mathbf{Z}^{2}$.

- Case $q=0$. Similar.

- Case $u / v=i$. Let $u^{\prime}=\bar{u}$. Then $u^{\prime}\langle n(\hat{T})\rangle=\bar{u} p u=p \in \mathbf{Z}$ and $u^{\prime} i\langle d(\hat{T})\rangle=\bar{u} i q v=\bar{u} u q=q \in \mathbf{Z}$. Thus $(p, q) \in \Psi(T) \cap \mathbf{Z}^{=} 2$.

- Case $u / v=-i$. Similar.

Proof of 2: Suppose that $\hat{T}$ is a diagram of $T$ and both $(p, q)$ and $\left(p^{\prime}, q^{\prime}\right)$ are elements of $f(T)=\Psi(T) \cap \mathbf{Z}^{2}=\Psi(\hat{T}) \cap \mathbf{Z}^{2}$. Then there exist 
elements $u$ and $u^{\prime}$ of $\Phi$ such that $p=u\langle n(\hat{T})\rangle, q=u i\langle d(\hat{T})\rangle, p^{\prime}=$ $u^{\prime}\langle n(\hat{T})\rangle$, and $q^{\prime}=u^{\prime} i\langle d(\hat{T})\rangle$.

- Case $\langle n(\hat{T})\rangle=0$. Then $p=p^{\prime}=0$. If $q=0$ then $\langle d(\hat{T})\rangle=0$, which implies that $q^{\prime}=0$. So $[p, q]=\left[p^{\prime}, q^{\prime}\right]=[0,0]$ and we are done. If $q \neq 0$, then $\langle d(\hat{T})\rangle \neq 0$. We then have $q^{\prime} / q=u^{\prime} i / u i \in$ $\mathbf{Q} \cap \Phi=\{ \pm 1\}$, so that $q= \pm q^{\prime}$. Hence $\left[p^{\prime}, q^{\prime}\right]=[0, \pm q]=[p, q]$, and we are done.

- Case $\langle n(\hat{T})\rangle \neq 0$.

Then $u^{\prime}\langle n(\hat{T})\rangle \neq 0$. We have $u / u^{\prime}=u\langle n(\hat{T})\rangle / u^{\prime}\langle n(\hat{T})\rangle=p / p^{\prime} \in$ $\Phi \cap \mathbf{Q}=\{ \pm 1\}$. If $u=u^{\prime}$ then $p=p^{\prime}$ and $q=q^{\prime}$. Thus $[p, q]=$ $\left[p^{\prime}, q^{\prime}\right]$. If $u=-u^{\prime}$ then $p=-p^{\prime}$ and $q=-q^{\prime}$. Again $[p, q]=\left[p^{\prime}, q^{\prime}\right]$ and we are done.

Proof of 3: If $\left(p^{\prime}, q^{\prime}\right) \neq(p, q)$ then $\left(p^{\prime}, q^{\prime}\right)=(-p,-q)$; replace $u$ with $-u \in \Phi$ as the coefficient of $\langle n(T)\rangle$ in $\Psi(T)$.

This completes the proof of the first claim in Theorem-and-Definition 1; the others follow.

Proposition 4. If $f(T)=p / q$ then $|p|$ and $|q|$ are the determinants $|\langle n(T)\rangle|$ and $|\langle d(T)\rangle|$, respectively.

Proof. We have that $(p, q) \in \Psi(T)$ so that for some $u$ in $\Phi$, $p=u\langle n(T)\rangle$ and $q=u i\langle d(T)\rangle$. So $|p|=|u\langle n(T)\rangle|=|\langle n(T)\rangle|$ and $|q|=$ $|u i\langle d(T)\rangle|=|\langle d(T)\rangle|$ as claimed.

\section{Properties of the Invariant}

Proposition 5. Additivity. If $T$ and $T^{\prime}$ are tangles then $f\left(T+T^{\prime}\right)=f(T)+$ $f\left(T^{\prime}\right)$, where the addition on the left-hand side of this equation is defined near the end of Section 2 and the addition on the right-hand side is defined in Section 6.

Proof. Let $T$ and $T^{\prime}$ be tangle diagrams. We have: 


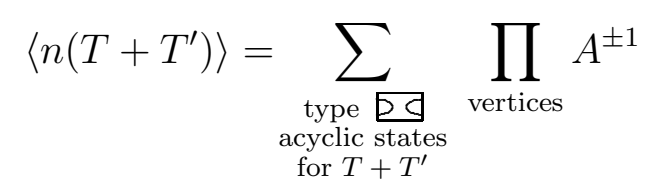

$$
\begin{aligned}
& =\sum_{\substack{\text { type } \sqrt{\square} \\
\text { acyclic states }}} \prod_{\text {vertices }} A^{ \pm 1}+\sum_{\substack{\text { type } \\
\text { acyclic states }}} \prod_{\text {vertices }} A^{ \pm 1} \\
& \left\langle d\left(T+T^{\prime}\right)\right\rangle=\sum_{\begin{array}{c}
\text { type } \\
\text { acyclic states } \\
\text { for } T+T^{\prime}
\end{array}} \prod_{\text {vertices }} A^{ \pm 1} \\
& =\sum_{\substack{\text { type } \\
\text { acyclic states }}} \prod_{\text {vertices }} A^{ \pm 1}
\end{aligned}
$$

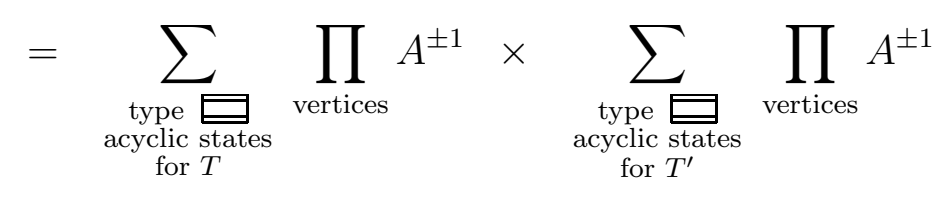

$$
\begin{aligned}
& =\langle d(T)\rangle\left\langle d\left(T^{\prime}\right)\right\rangle
\end{aligned}
$$

In equations (2) and (3) we are summing over acyclic states of the given homotopy class. States for a sum of two tangle diagrams correspond to pairs of states- one for each tangle. The double boxes indicate the type of this pair. 


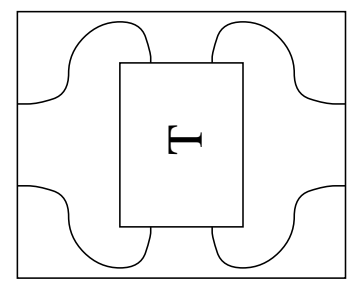

FiguRe 7. Rotation of a tangle $T$

Suppose that $T$ and $T^{\prime}$ take the values $[p, q]$ and $\left[p^{\prime}, q^{\prime}\right]$, respectively. Then there exist $u, u^{\prime} \in \Phi$ such that $p=u\langle n(T)\rangle, q=u i\langle d(T)\rangle, p^{\prime}=$ $u^{\prime}\left\langle n\left(T^{\prime}\right)\right\rangle$, and $q^{\prime}=u^{\prime} i\left\langle d\left(T^{\prime}\right)\right\rangle$. Substituting in equation (2), we have

$$
\left\langle n\left(T+T^{\prime}\right)\right\rangle=p \bar{u}\left(-i q^{\prime} \overline{u^{\prime}}\right)+-i q \bar{u} p^{\prime}\left(\overline{u^{\prime}}\right)=-i \bar{u} \overline{u^{\prime}}\left(p q^{\prime}+p^{\prime} q\right) .
$$

and substituting in equation (3),

$$
\left\langle d\left(T+T^{\prime}\right)\right\rangle=(-i q \bar{u})\left(-i q^{\prime} \overline{u^{\prime}}\right)=-q q^{\prime} \bar{u} \overline{u^{\prime}} .
$$

Multiplying (4) and (5) by iuu yields

$$
i u u^{\prime}\left\langle n\left(T+T^{\prime}\right)\right\rangle=p q^{\prime}+p^{\prime} q
$$

and

$$
\left(i u u^{\prime}\right) i\left\langle d\left(T+T^{\prime}\right)\right\rangle=\left(i u u^{\prime}\right) i\left(-q q^{\prime} \bar{u} \overline{u^{\prime}}\right)=q q^{\prime} .
$$

Thus, since $i u u^{\prime} \in \Phi$, the pair $\left(p q^{\prime}+p^{\prime} q, q q^{\prime}\right)$ belongs to the set $\Psi\left(T+T^{\prime}\right)$. The value of the invariant is $\left(p q^{\prime}+p^{\prime} q\right) / q q^{\prime}=p / q+p^{\prime} / q^{\prime}$. Proposition 5 follows.

Notation 4. If $T$ is a tangle, define $T^{*}$ as the tangle obtained by rotating $T$ by the angle $\pi / 2$ and redirecting the endpoints (see Figure 7 ).

Proposition 6. If $T$ takes the value $[p, q]$ then $T^{*}$ takes the value $[-q, p]$. 


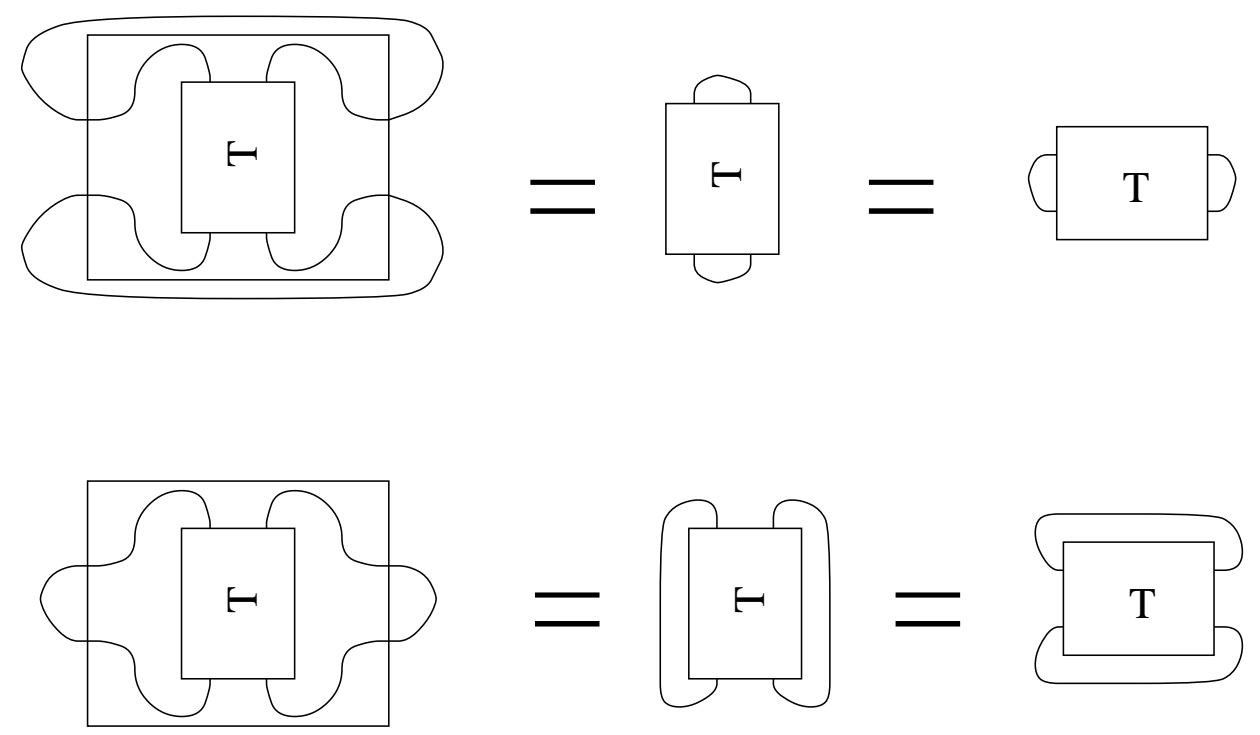

FiguRE 8. Isotopy of numerator and denominator closures

Proof. First note that given a diagram for $T$ Figure 7 yields a diagram for $T^{*}$. Let us fix a diagram $D$ for $T$ and let $D^{*}$ be the corresponding diagram for $T^{*}$.

The first line of Figure 8 shows that $n\left(D^{*}\right)$ is regular isotopic to $d(D)$ and the second line shows that $d\left(D^{*}\right)$ is regular isotopic to $n(D)$. Since $T$ takes the value $[p, q]$, the pair $(p, q) \in \Psi(D)$, ie. there exists a $u \in \Phi$ such that $p=u\langle n(D)\rangle$ and $q=i u\langle d(D)\rangle$, or $\langle n(D)\rangle=p \bar{u}$ and $\langle d(D)\rangle=q(-i \bar{u})$.

Set $u^{*}=i u \in \Phi$.

Then

$$
u^{*}\left\langle n\left(D^{*}\right)\right\rangle=u^{*}\langle d(D)\rangle=i u q(-i \bar{u})=q,
$$

and

$$
u^{*} i\left\langle d\left(D^{*}\right)\right\rangle=u^{*} i\langle n(D)\rangle=i u i p \bar{u}=-p .
$$

Thus $u^{*} \in \Phi$ implies that $(q,-p) \in \Psi\left(D^{*}\right)$; the value of the invariant is $[q,-p]=[-q, p]$. This completes the proof of Proposition 6 . 
Notation 5. In view of Proposition 6 we will introduce an operation labelled $*$ on $\mathbf{A}$, defined by $[p, q]^{*}=[-q, p]$. Then Proposition 6 asserts that $f\left(T^{*}\right)=f(T)^{*}$ for all tangles $T \in \mathbf{T}$.

Proposition 7. Reflecting a tangle $(J, T)$ about any of the three planes $x=$ $1 / 2, y=1 / 2$, or $z=1 / 2$ yields another tangle $(J, \bar{T})$ whose invariant is $-p / q$, where $p / q$ is the value of the invariant on $T$.

Proof. Let $D$ be a diagram for the tangle $T$, and $\bar{D}$ be the reflection of $D$ through the given plane. If $S$ is a state for $D$ then $S$ may also be reflected through the given plane (in the case of the plane $z=1 / 2$ the state $S$ is left unchanged) yielding a state $\bar{S}$ for $\bar{D}$. Note that $\bar{S}$ is a numerator (denominator) state precisely if $S$ is. Therefore the terms in the expansion of the Kauffman bracket of $n(\bar{D})$ correspond precisely to those of $n(D)$ and similarly for $d(\bar{D})$ and $d(D)$. Since crossings for $D$ also correspond to crossings for $\bar{D}$ the factors of each term of the expansion for $\langle n(\bar{D})\rangle$ correspond precisely to those for $\langle n(D)\rangle$ and similarly for $\langle d(\bar{D})\rangle$ and $\langle d(D)\rangle$. But if $S$ is resolved as an $A$-state at a crossing of $D$ then $\bar{S}$ is resolved as an $A^{-1}$-state at the corresponding crossing of $\bar{D}$ and similarly with the roles of $A$ and $A^{-1}$ reversed. By $A^{-1}=\bar{A}$ (here $\bar{A}$ is the complex conjugate of $A$ ) and the fact that the Kauffman bracket is a polynomial in $A$ with real (in fact integer) coefficients we have that $\langle n(\bar{D})\rangle=\overline{\langle n(D)\rangle}$ and $\langle d(\bar{D})\rangle=\overline{\langle d(D)\rangle}$.

As before, write $\langle n(D)\rangle=p \bar{u}$ and $\langle d(D)\rangle=q(-i \bar{u})$ for some $u \in \Phi$.

We have

$$
\begin{gathered}
\langle n(\bar{D})\rangle=\overline{\langle n(D)\rangle}=\overline{p \bar{u}}=p u \\
\langle d(\bar{D})\rangle=\overline{\langle d(D)\rangle}=\overline{-i q \bar{u}}=q u i .
\end{gathered}
$$

Set $u^{\prime}=-\bar{u}$. Then

$$
u^{\prime}\langle n(\bar{D})\rangle=-\bar{u} p u=-p
$$


AN OBSTRUCTION TO EMBEDDING 4-TANGLES IN LINKS

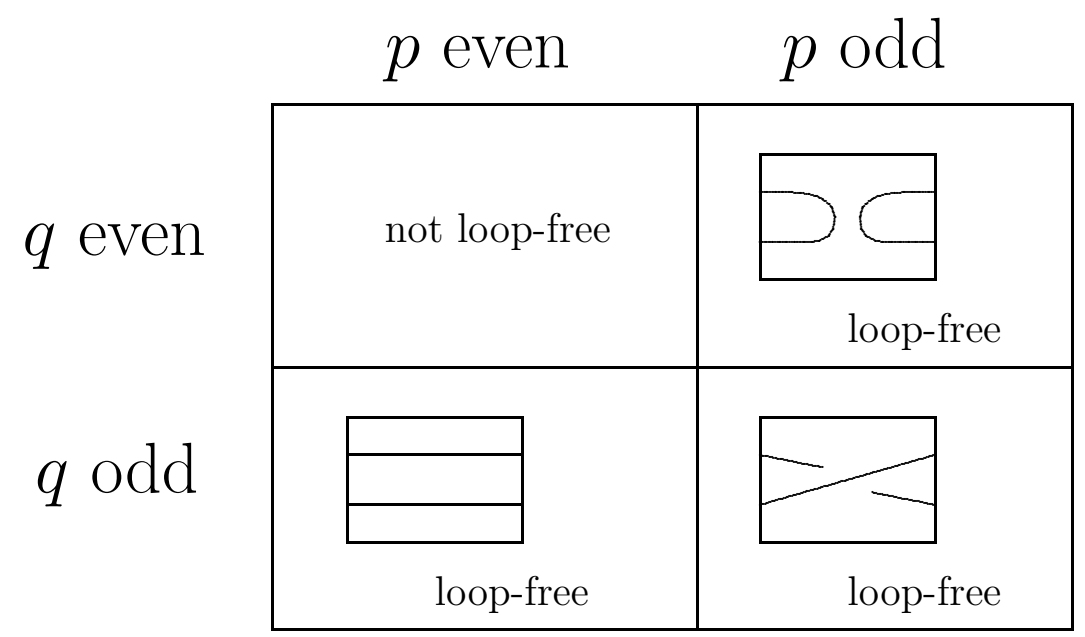

FiguRE 9. Homotopy class and parity of $p$ and $q$

and

$$
u^{\prime} i\langle d(\bar{D})\rangle=-\bar{u} i(q u i)=q .
$$

Thus $u^{\prime} \in \Phi$ implies that $(-p, q) \in \Psi(\bar{D})$. The value of the invariant on the tangle $\bar{T}$ is $[-p, q]$. This is Proposition 7 .

Proposition 8. If $\langle L\rangle=p u$, with $p \in \mathbf{Z}$ and $u \in \Phi$, then $p$ (or the determinant $|p|)$ is even if and only if $L$ has more than one component.

Proof. It will be shown in Section 11 that $p= \pm V_{L}(-1)$, where $V$ is the Jones polynomial. The result now follows from [2, Thm. 15, p. 107] noting that $\left.\pm V_{L}(-1) \equiv V_{L}(1) \bmod 2\right)$.

From Proposition 4 we now have:

Proposition 9. For a 4-tangle $T$ with invariant $[p, q]$ the parities of $p$ and $q$ are related to the homotopy class of $T$ according to Figure 9.

Here the diagrams in the three lower and right-hand entries describe the homotopy class (Definition 1) of the tangle. The reader may wish to corroborate Figure 9 against Figure 13 and the values of the invariant given there. 


\section{The Embeddability Condition}

We say that the tangle $T$ can be embedded in or sits inside the link $L$ if there is a representative of $L$ whose intersection with the unit cube $J$ is the 1-manifold $T$.

The main result of this paper is the following:

Theorem 1. Suppose that the tangle $T$ can be embedded in the link $L$.

Version 1. If $f(T)=p / q$ then $\operatorname{gcd}(p, q)||\langle L\rangle \mid$ as integers.

Version 2. If $n(T)$ and $d(T)$ are the links of Figure 1, then

$$
\operatorname{gcd}(|\langle n(T)\rangle|,|\langle d(T)\rangle|)||\langle L\rangle \mid
$$

as integers.

Since the determinant of the unknot is 1 , we have

Corollary 1. The tangle $T$ cannot sit inside the unknot unless the determinants $|\langle n(T)\rangle|$ and $|\langle d(T)\rangle|$ of the links $n(T)$ and $d(T)$ are relatively prime.

The proof of the theorem uses the following lemma:

Lemma 5. If the tangle $T$ can be embedded in the link $L$, then there is a second tangle $T^{\prime}$ such that $L$ is ambient isotopic to the link $n\left(T+T^{\prime}\right)$.

Proof of Lemma 5. The tangle $T$ is a properly embedded 1manifold in a topological 3-ball (actually a cube) $J$ contained in $S^{3}$ with $T \cap \partial J$ consisting of 4 points. Let $B$ denote the closure of $S^{3} \backslash J$, also a 3-ball. Then the intersection of $L$ with $B$ is another tangle, that we can call $T^{\prime}$. Since $T^{\prime}$ is not space-filling, it is clear that $T^{\prime}$ can be brought into a cube in $\mathbf{R}^{\mathbf{3}}$ adjacent to the cube $J$ by an ambient isotopy that is the identity on the boundary of $B$. This produces the standard picture in the form $n\left(T+T^{\prime}\right)$.

Proof of Theorem 1. Version 1: Let $T^{\prime}$ be the tangle given by Lemma 5. Write $f\left(T^{\prime}\right)=[r, s]$. Then $f\left(T+T^{\prime}\right)=[p s+q r, q s]$ and so by 

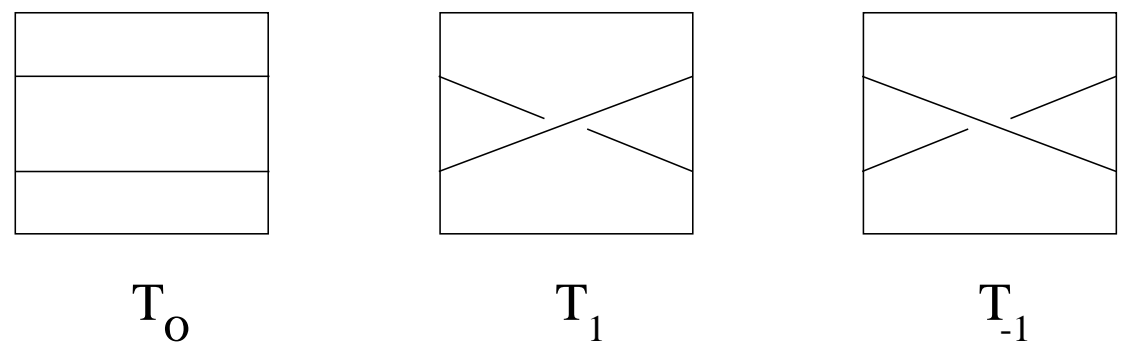

FiguRE 10. Three very simple tangles

Proposition 4, $|p s+q r|=\left|\left\langle n\left(T+T^{\prime}\right)\right\rangle\right|=|\langle L\rangle|$ and

$$
\operatorname{gcd}(p, q)|| p s+q r|=|\langle L\rangle \mid
$$

as claimed.

Theorem 1, Version 2 follows from Proposition 4 and Theorem 1, Version 1.

\section{Sample Calculations}

For the tangle $T_{0}$ depicted in Figure 10, $n\left(T_{0}\right)$ is the unlink of two components and $d\left(T_{0}\right)$ is the unknot. Let $u=-i$. Then $u\left\langle n\left(T_{0}\right)\right\rangle=-i \cdot 0=$ $0 \in \mathbf{Z}$ and $u i\left\langle d\left(T_{0}\right)\right\rangle=\left\langle d\left(T_{0}\right)\right\rangle=1 \in \mathbf{Z}$. The value of the invariant on $T_{0}$ is $0 / 1$.

Now consider the tangle $T_{1}$ of Figure 10 . We have that $n\left(T_{1}\right)$ and $d\left(T_{1}\right)$ are both unknots and by applying the skein relation for the Kauffman bracket as in equation (1), $\left\langle n\left(T_{1}\right)\right\rangle=A$ and $\left\langle d\left(T_{1}\right)\right\rangle=A^{-1}$. Set $u=\bar{A}=e^{-\pi i / 4}$. Then $u\left\langle n\left(T_{1}\right)\right\rangle=e^{-\pi i / 4} e^{\pi i / 4}=1 \in \mathbf{Z}$ and $u i\left\langle d\left(T_{1}\right)\right\rangle=$ $e^{-\pi i / 4} i e^{-\pi i / 4}=1 \in \mathbf{Z}$. Thus the value of the invariant on $T_{1}$ is $1 / 1$.

The value of the invariant on $T_{-1}$ of Figure 10 is $1 /-1=-1 / 1$. This value may be found by noticing that $T_{-1}$ can be obtained from $T_{1}$ by either a rotation (Proposition 6) or a reflection (Proposition 7). Alternatively one may find this value by solving for $f\left(T_{-1}\right)$ in the formal fraction equation $1 / 1+f\left(T_{-1}\right)=0 / 1$ which results from applying additivity to the tangle equation $T_{1}+T_{-1}=T_{0}$. 


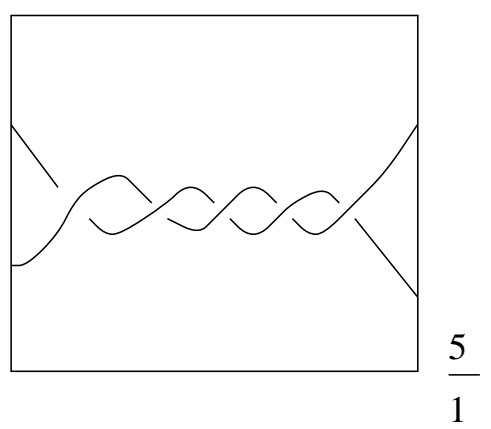

Figure 11. An integral tangle

Definition 3. Let $n \in \mathbf{Z}$; we will define the tangle $T_{n}$. $T_{n}$ has been defined above for $n \in\{-1,0,1\}$. For $n>1$ define $T_{n}$ as the sum of $n$ copies of $T_{1}$; for $n<-1$ define $T_{n}$ as the sum of $|n|$ copies of $T_{-1}$. We will call $T_{n}$ for $n \in \mathbf{Z}$ an integral tangle as in [3]. By the additivity of the invariant and the associativity of the monoid structure on $\mathbf{A}, T_{n}$ is assigned the value $[n, 1]=n / 1 . T_{5}$ is depicted in Figure 11.

Proposition 10. Define the function $\phi: \mathbf{Z} \rightarrow \mathbf{A}: n \mapsto n / 1$. Then we have that $T_{m}+T_{n}=T_{m+n}$ for all $m, n \in \mathbf{Z}$ and that $\phi^{-1} \circ f: T_{n} \mapsto n$ is a well-defined group isomorphism from the submonoid of integral tangles to the integers.

Proof. The proof is entirely algebraic except for the fact that $T_{n}+$ $T_{-n}=T_{0}$, by successive applications of the Reidemeister type II move, and is left to the interested reader.

Example 1. "Square knot" tangle.

The tangle $\left(T_{3}\right)^{*}+\left(T_{-3}\right)^{*}$, commonly known as a square knot, is drawn on the left side of Figure 12. The value of the invariant (see Proposition 6 and Notation 5) is:

$$
\left(\frac{3}{1}\right)^{*}+\left(\frac{-3}{1}\right)^{*}=\frac{-1}{3}+\frac{1}{3}=\frac{0}{9} .
$$

Note here that $\operatorname{gcd}(0,9)=9$, so this tangle does not sit inside an unknot or for example a trefoil (determinant $=3$ ). 


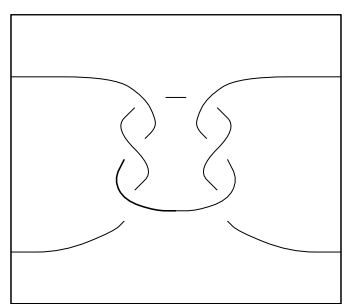

square

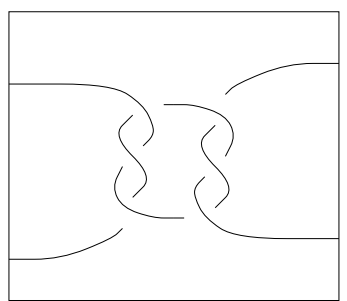

granny

Figure 12. Two commonly confused tangles

Example 2. A "granny knot" tangle $G$ is depicted on the right side of Figure 12 .

$$
\begin{gathered}
G=\left(T_{3}\right)^{*}+\left(T_{3}\right)^{*} ; \\
f(G)=\left(\frac{3}{1}\right)^{*}+\left(\frac{3}{1}\right)^{*}=\frac{-1}{3}+\frac{-1}{3}=\frac{-6}{9} .
\end{gathered}
$$

Here $\operatorname{gcd}(-6,9)=3$, so $G$ possibly sits inside a trefoil ${ }^{1}$ but not, for instance, an unknot or a Hopf link (determinant $=2$ ).

Figure 13 gives the value of the invariant for various other tangles.

\section{Determinant}

The non-negative integer $|\langle L\rangle|$ has many other formulations. Firstly, it is, up to sign, the value at $t=-1$ of the Jones polynomial $V_{L}(t)$ : This is because to compute the Jones polynomial from the Kauffman bracket we replace $A^{-4}$ with $t$ and multiply by a power of $-A^{-3}$ which has absolute value 1 for our choice of $A$. Since $A^{-4}=-1$, setting $t=-1$ in the Jones polynomial will give, in absolute value, the same quantity as the Kauffman bracket. But the Jones polynomial has integer coefficients, so both $V_{L}(-1)$ and $|\langle L\rangle|$ are integers. Therefore they agree up to sign. Secondly, when $L$ is a knot $|\langle L\rangle|$ is the value at -1 of the Alexander polynomial (see [2, Cor. 13 ,

\footnotetext{
${ }^{1}$ In fact John Wood has pointed out to the author that it does: $n\left(G+T_{1}\right)=n\left(T_{-3}\right)$, a right-handed trefoil.
} 

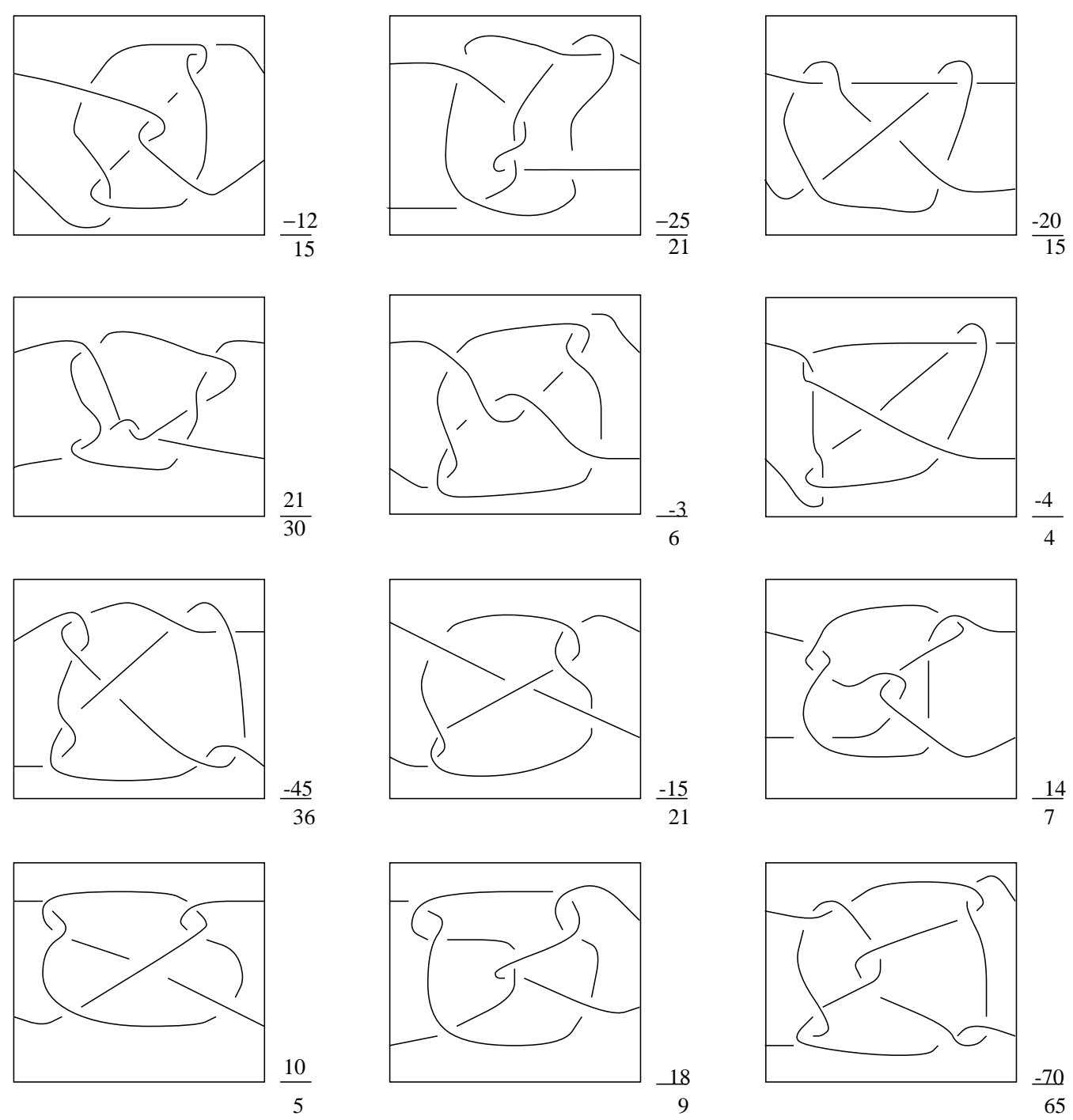

FIGURE 13. Some tangles and their associated values.

p. 107]). Finally, when $L$ is a knot $|\langle L\rangle|$ is the order of the first homology of the two-fold cover of $S^{3}$ branched over $L$ (see [4]).

The integer $|\langle L\rangle|$ is known classically as the determinant of the link $L$.

Remark. Probably the easiest way to systematically calculate the value of the determinant on a large number of links with few crossings (at most ten, say) is by calculating the value of the invariant on a collection of 
related tangles. For instance we were able to use state summations for the Kauffman bracket to evaluate the invariant on each of the twelve tangles in Figure 13. This gives the value of the determinant for $2 \cdot 12=24$ links representing at least 18 (the number of positive integers which appear up to sign in the formal fractions) link types, each with no more than ten crossings.

For determinants of some simple individual links, the reader is offered two strategies:

1) For algebraic links- links arising as $n(T)$ for some algebraic tangle $T$ (see Section 12) such as the square or granny tangle- calculate the determinant as the absolute value of the numerator of the invariant associated to the tangle, which can be computed using the additivity, rotation and reflection formulae (Section 8).

2) Consult the tables of knot- and link-diagrams in [4] (where it is explained how to calculate the determinant from the data accompanying each link-diagram).

\section{ReAlizaBility}

Let $\mathbf{T}_{\mathbf{0}} \subset \mathbf{T}$ be the set of algebraic tangles with unknotted components, where the set of "algebraic" tangles is the closure under addition, rotation and reflection of the set of integral tangles as in [3], and "unknotted components" means that each component of $T$ is unknotted (for the two arc components this means that the knot formed by adjoining an arc in $\partial J$ with the same endpoints is an unknotted loop).

We will show

Theorem 2. The function $f$ maps $\mathbf{T}_{\mathbf{0}}$ onto $\mathbf{A}$.

This defines a large (infinite) class of tangles for which Theorem 1 gives non-trivial information.

Proof of Theorem 2. Let $[p, q] \in \mathbf{A}$, and write $p=2^{n} d p^{\prime}, q=$ $2^{n} d q^{\prime}$, where $n \geq 0, d$ is odd, and $p^{\prime}$ and $q^{\prime}$ are relatively prime. Note that if $q^{\prime}$ is not odd, then $p^{\prime}$ is odd. If we can show that the value $[q, p]$ is achieved 
on $\mathbf{T}_{\mathbf{0}}$ then by rotating and reflecting (section 8 ), we arrive at a tangle, still in $\mathbf{T}_{\mathbf{0}}$, which is mapped to $[p, q]$. Thus we may assume that $q^{\prime}$ is odd.

We have

$$
\frac{p}{q}=\frac{2^{n} d p^{\prime}}{2^{n} d q^{\prime}}=\frac{0}{2^{n}}+\frac{0}{d}+\frac{p^{\prime}}{q^{\prime}}
$$

We will explicitly find tangles in $\mathbf{T}_{\mathbf{0}}$ whose values are each of the three terms in the rightmost expression of (6) and then show that their sum also lies in $\mathbf{T}_{\mathbf{0}}$.

First, we describe the tangle on which $f$ takes value $\left[p^{\prime}, q^{\prime}\right]$ :

Expand the rational number $p^{\prime} / q^{\prime}$ as a finite continued fraction. Recall that a finite continued fraction is obtained by starting with the integer 0 and alternately applying for a finite number of iterations the operations of a) addition of a non-zero integer $k$, (not necessarily the same integer $k$ for different iterations) and b) taking the reciprocal $r \mapsto 1 / r$. Corresponding to these algebraic operations are the topological operations of $\left.\mathrm{a}^{\prime}\right)$ the addition of an integral tangle $T_{k}, k \neq 0$, and $\mathrm{b}^{\prime}$ ) a rotation and a reflection (see Section 8).

We notice that i) the invariant on tangle $T_{0}$ is 0 and ii) an operation of type $\mathrm{a}^{\prime}\left(\mathrm{b}^{\prime}\right.$, respectively) on a tangle $T$ has the effect of performing an operation of type a (b, respectively) on the invariant of $T$. Thus we have only to expand the rational number $p^{\prime} / q^{\prime}$ as a continued fraction and then replace the operations a) and b) with the operations $\mathrm{a}^{\prime}$ ) and $\mathrm{b}^{\prime}$ ) (starting from the tangle $T_{0}$ ) to arrive at tangle whose invariant is $p^{\prime} / q^{\prime}$. A tangle constructed in such a way is called a rational tangle (see [3]). By virtue of this mode of construction, rational tangles are algebraic and have unknotted strands (there are no loop components); ie. lie in $\mathbf{T}_{\mathbf{0}}$.

Next, we describe below the tangle on which $f$ takes the value $[0, d]$. Let $d^{\prime}$ be the integer (not the formal fraction) $(d-1) / 2$. Consider the following calculation: 


$$
\begin{gathered}
\frac{1}{d}+\frac{1}{d}=\frac{2 d}{d^{2}} \\
\left(\frac{2 d}{d^{2}}\right)^{*}=\frac{-d^{2}}{2 d} \\
\frac{-d^{2}}{2 d}+\frac{d^{\prime}}{1}=\frac{-d}{2 d} \\
\left(\frac{-d}{2 d}\right)^{*}=\frac{2 d}{d} \\
\frac{2 d}{d}+\frac{-2}{1}=\frac{0}{d} .
\end{gathered}
$$

Corresponding to these algebraic operations are the following topological ones: We start with a variant of the granny $(d=3)$ tangle and alternately add integral tangles and rotate. These operations preserve the properties that the tangle is algebraic and that both strands (there are no loop components since $d$ is odd) are unknotted. By the properties of the invariant described in Section 8, the resulting tangle $T$ satisfies $f(T)=0 / d$.

Here is an explicit formula for $T$ of the above paragraph:

$$
\left(\left(\left(T_{-d}\right)^{*}+\left(T_{-d}\right)^{*}\right)^{*}+T_{d^{\prime}}\right)^{*}+T_{-2} .
$$

Finally, define the tangle $S$ as $\left(T_{2}^{*}+T_{0}^{*}\right)^{*}$, depicted in Figure 14 . Then

$$
f(S)=\left(\left(\frac{2}{1}\right)^{*}+\left(\frac{0}{1}\right)^{*}\right)^{*}=\left(\frac{-1}{2}+\frac{1}{0}\right)^{*}=\left(\frac{2}{0}\right)^{*}=\frac{0}{2} .
$$

The sum of $n$ copies of $S$ lies in $\mathbf{T}_{\mathbf{0}}$ and has invariant

$$
\overbrace{\frac{0}{2}+\cdots+\frac{0}{2}}^{n \text { times }}=\frac{0}{2^{n}} .
$$

It remains to be seen that the sum of these three tangles, all in $\mathbf{T}_{\mathbf{0}}$, also lies in $\mathbf{T}_{\mathbf{0}}$. 


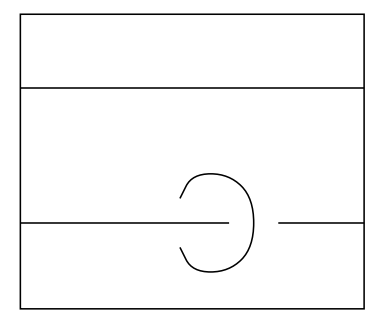

Figure 14. The tangle $S$ used to show realizability of formal fractions

Since adding multiple copies of the tangle $S$ preserves both the properties of being algebraic and having unknotted components, we will be done proving Theorem 2 if we can show that the sum of the two tangles corresponding to the last two terms in (6) is in $\mathbf{T}_{\mathbf{0}}$. For this we need the following lemma:

Lemma 6. i) Let $T$ be a tangle mapped to some element $[p, q]$ of $\mathbf{A}$. Then $q$ is odd precisely if $T$ is loop-free and the homotopy class of $T$ is in the set

$$
H=\{\boxminus, \bigotimes\}
$$

ii) Consider a pair of tangles with the following properties: algebraic, unknotted components, loop-free, and homotopy class in $H$. Then their sum also has these properties.

Proof of Lemma 6. Part i): See Proposition 9. Part ii): Obvious. The sum can be seen to have the last three properties by examining its components one at a time.

Proof of Theorem 2 (resumed). We have two algebraic tangles with unknotted components, one of which is mapped to $[0, d]$ and the other of which is mapped to $\left[p^{\prime}, q^{\prime}\right]$. Note that the denominators $d$ and $q^{\prime}$ are both odd. Therefore Lemma 6 part i) applies to both of these tangles. Lemma 6 part ii) then asserts that their sum is also algebraic with unknotted components, ie. belongs to $\mathbf{T}_{\mathbf{0}}$ as claimed. 
This completes the proof of Theorem 2 .

Remark. In the construction of the rational tangle with invariant $\left[p^{\prime}, q^{\prime}\right]$ above there are many ways of expanding the rational number $p^{\prime} / q^{\prime}$ as a continued fraction. Furthermore, in the construction of the rational tangle itself we may add the integral tangle either on the left or on the right, we may rotate through an angle either of $\pi / 2$ or of $-\pi / 2$ (which may be shown to have the same effect on the invariant) and we may reflect about any of the planes $x=1 / 2, y=1 / 2$, or $z=1 / 2$. Thus our procedure gives many distinct tangle diagrams associated to the same value of the invariant. However Goldman and Kauffman prove in $[6]$ that the rational tangles represented by these diagrams are all ambient isotopic to each other.

\section{Fox $n$-COLOURINGS}

Daniel Silver has pointed out to the author that all of the tangles in Figure 13 with $\operatorname{gcd}(p, q)$ odd and not equal to 1, as well as the square and granny tangles, can alternatively be shown by Fox $n$-colourings not to be embeddable in unknots. (For the idea, without the terminology, of a Fox $n$-colouring see Chapter 10 of [7]; [8] contains an up-to-date review of this concept.) One need only produce a non-trivial $n$-colouring of a diagram for the tangle in which all of the overcrossing arcs incident to one (or more) of the four endpoints are coloured the same, say with the label ("colour") $a$. Fox $n$-colourings of this type with $n=3$ ("tricolourings") for the square and the granny tangles are depicted in Figure 15. If the tangle diagram could be embedded in an unknot diagram then by colouring all the remaining arcs (outside the tangle diagram) with the same label $a$, we would have a non-trivial colouring of the unknot, a contradiction.

If both the numerator and the denominator closure of a 4-tangle $T$ can be $n$-coloured non-trivially, can the tangle be non-trivially $n$-coloured so that all four endpoints get the same colour? It is known that a knot with determinant $p$ can be non-trivially $q$-coloured, where $q$ is a prime, if 


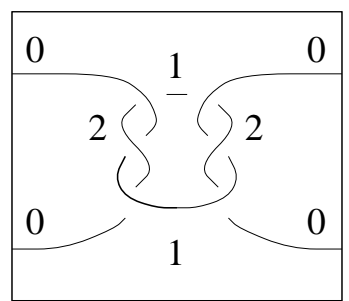

square

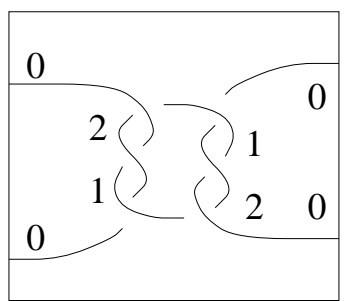

granny

FiguRE 15. Tricolourings which show neither square nor granny tangle embeds in an unknot

and only if $q$ divides $p$. (See [9], p. 45.) If the question above were true then we would have the following situation: If there were a prime $q$ dividing both the determinant of $n(T)$ and of $d(T)$ then the tangle $T$ would have a non-trivial $q$-colouring in which all four endpoints receive the same colour and thus could not sit inside an unknot. This would provide an alternate proof of Corollary 1.

\section{Generalizations And Further Questions}

For any positive even integer $p$, choose a fixed set $E$ of $p$ points in $S^{2}=\partial B$ where $B$ is the unit ball in $\mathbf{R}^{3}$. Define a $p$-tangle as a onedimensional properly embedded tame subspace of $B$ whose boundary is $E$. By connecting the endpoints in a trivial way, a pair $T$ and $T^{\prime}$ of $p$-tangles gives rise to a link $T \# T^{\prime}$. For $p=8 T \# T^{\prime}$ is drawn in Figure 16.

Is there a finite collection $\mathbf{S}_{\mathbf{p}}$ of $p$-tangles with the following property? For any tangle $T$ and integer $d$ such that $d||\langle T \# S\rangle \mid$ for all $p$-tangles $S \in \mathbf{S}_{\mathbf{p}}$, we have that $d||\langle L\rangle \mid$ for any link $L$ which intersects the unit ball $B$ in the one-dimensional subspace $T$.

If $p=4$ this question is answered by the present paper in the affirmative: The set $\mathbf{S}_{4}$ can be chosen to consist of two elements $T_{0}$ and $T_{0}^{*}$ such that $T \# T_{0}$ is ambient isotopic to $n(T)$ and $T \# T_{0}^{*}$ is ambient isotopic to $d(T)$. The result now follows from Theorem 1 version 2 . 


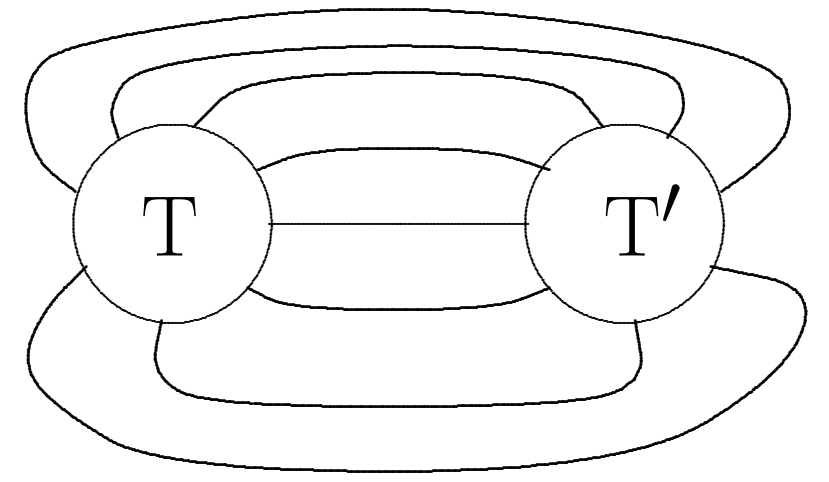

Figure 16. A connected sum of two 8-tangles $T$ and $T^{\prime}$

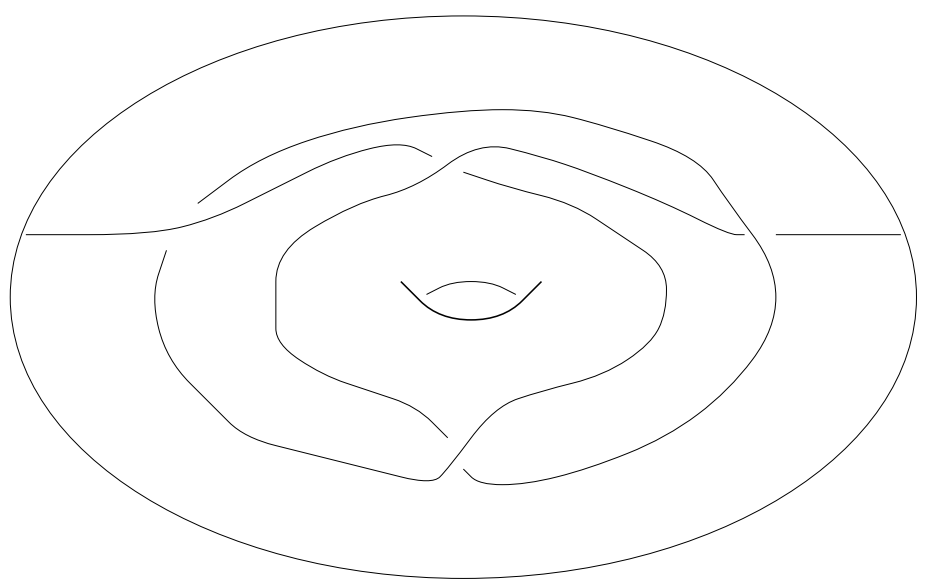

FiguRE 17. A properly embedded arc in a solid torus

It is natural to ask how a link may intersect subsets of $S^{3}$ which are not balls. For instance, what links can intersect a solid torus in the arc shown in Figure 17? A little experimentation shows that this configuration sits inside a trefoil (determinant $=3)$, a figure-eight knot (determinant $=5$ ), and a 2,7 torus knot (determinant $=7$ ). Does it sit inside an unknot?

\section{ACKNOWLEDGMENTS}

I would like to thank my doctoral thesis advisor Peter B. Shalen for his patient guidance with the compiling of the thesis which resulted in this paper. It was Peter who first recognized the generality of my methods 
beyond the case of the link in question being an unknot and who suggested the "intersection with cube" formulation of embeddability. In addition I am deeply grateful to Lou Kauffman for pointing out to me the fraction addition formula of John H. Conway and for various other suggestions which helped to streamline this paper. Finally, thanks also to Sandy Rutherford for assistance with $\mathrm{IAT}_{\mathrm{E} X}$.

\section{REFERENCES}

[1] Kauffman, L.: State models and the Jones polynomial. Topology, 26(3):395-407, 1987.

[2] Jones, V.: A polynomial invariant for knots via von Neumann algebras. Bull. Amer. Math. Soc., 12(1):103-112, 1985.

[3] Conway, J.: An enumeration of knots and links and some of their algebraic properties. In Computational Problems in Abstract Algebra, ed. J. Leech, pages 329-358. New York, Pergammon Press, 1970.

[4] Rolfsen, D.: Knots and Links. Berkeley, CA, Publish or Perish, 1976.

[5] Kauffman, L.: Knots and Physics. Teaneck, NJ, World Scientific, 1991.

[6] Goldman, J. and Kauffman, L.: Rational tangles. Advances in Applied Mathematics, 18:300-332, 1997.

[7] R.H. Fox: A quick trip through knot theory. In Topology of 3-Manifolds and Related Topics, ed. M.K. Fort, pages 120-167. New Jersey, Prentice-Hall, 1961.

[8] Silver, D. and Williams, S.: Generalized $n$-colorings of links. Proceedings of 1995 Conference in Knot Theory, Banach Center Publications, Vol. 42, 1998.

[9] Livingston, C.: Knot Theory. Carus Mathematical Monographs Vol. 24, Mathematical Association of America, 1993. 